\title{
A history of radio detection of cosmic-rays
}

DOI:

10.1093/astrogeo/aty149

Document Version

Final published version

Link to publication record in Manchester Research Explorer

\section{Citation for published version (APA):}

Spencer, R., \& Rapley, C. (2018). A history of radio detection of cosmic-rays. Astronomy \& Geophysics, 59(3), 3.33-3.37. https://doi.org/10.1093/astrogeo/aty149

\section{Published in:}

Astronomy \& Geophysics

\section{Citing this paper}

Please note that where the full-text provided on Manchester Research Explorer is the Author Accepted Manuscript or Proof version this may differ from the final Published version. If citing, it is advised that you check and use the publisher's definitive version.

\section{General rights}

Copyright and moral rights for the publications made accessible in the Research Explorer are retained by the authors and/or other copyright owners and it is a condition of accessing publications that users recognise and abide by the legal requirements associated with these rights.

\section{Takedown policy}

If you believe that this document breaches copyright please refer to the University of Manchester's Takedown Procedures [http://man.ac.uk/04Y6Bo] or contact uml.scholarlycommunications@manchester.ac.uk providing relevant details, so we can investigate your claim.

\section{open 2 Access}




\section{A history of radio \\ detection of cosmic-rays}

\author{
Ralph Spencer and Chris \\ Rapley explore the role played \\ by Jodrell Bank in early attempts \\ to detect cosmic rays - and how \\ the technique is now becoming \\ more widely used.
}

\section{he question of the origin of cos- mic rays has dominated efforts in astroparticle research ever since} their discovery by Victor Hess in 1911. Although likely sources at low energies are partly understood, the origin of the highest energy cosmic rays has remained a mystery. The basic problem is that highenergy events are rare, with cosmic rays of more than $10^{20} \mathrm{eV}$ expected at a rate of 1 per $\mathrm{km}^{2}$ of the Earth's surface per century. Thus a detector covering a large area - hundreds of $\mathrm{km}^{2}$-is needed in order to study reasonable numbers of events. High-energy interactions in the Earth's atmosphere produce many secondary particles in an extensive air shower before reaching the Earth's surface. Even so, plenty of particle detectors are required because the characteristic width of the shower is only $\sim 80 \mathrm{~m}$ for the electromagnetic cascade and $\sim 320 \mathrm{~m}$ for the muon component (Patrignani et al. 2006). These facts led Blackett and Lovell (1941) to suggest that it may be possible to detect distant showers by means of the radar echoes from the trail of ionization left behind the shower front as it passes though the atmosphere.

Early experiments in 1946 took place in the Cheshire fields at Jodrell Bank, acquired by the University of Manchester as a botanical station. They used surplus military radars but failed to detect cosmic rays. Instead they found high-altitude trails of meteors and the work led to the rapid development of radio astronomy at Jodrell Bank (Lovell 1968). The reason for the non-detection was damping caused by the rapid capture of free electrons by neutral atoms and molecules in the lower atmosphere; hence the radar reflective trail from a passing cosmic ray has only a fleeting existence, lasting less than 100ns. Various experiments have been proposed since (e.g. Gorham 2001), though none has proved successful at unequivocally detecting cosmic-ray air showers by radar.

The passive radio story is, however, quite different. This technique has now become a useful addition to air shower studies, after a period of low activity from the early 1970s. Modern digital technology led to a revival in the 2000s (Falcke \& Gorham 2003) and there has been a flurry of activity since, with major developments in understanding the emission mechanisms and the radio characteristics, together with new experimental techniques. It now seems appropriate to discuss some of the early work at Jodrell Bank before the remaining protagonists forget.

\section{The original idea}

The possibility that the secondary particle shower itself may emit at radio wavelengths was first suggested by John Jelley in his book on Cherenkov radiation (Jelley 1958). It was thought that the radio emission could possibly extend over a greater area than the particles over the ground, and that a large area could be covered relatively inexpensively. Early measurement at microwave frequencies proved unsuccessful. However, Askaryan (1962) suggested that the electromagnetic shower could contain an excess of electrons, caused by the inflight annihilation of positrons. Because the electrons are relativistic, moving through the Earth's atmosphere where the velocity of light is less than $c$, and concentrated in a thin ( $2 \mathrm{~m}$ depth) shower front, then they could emit coherent Cherenkov radiation at wavelengths of a few metres. So perhaps observations at a few $10 \mathrm{~s}$ of $\mathrm{MHz}$ would be successful after all. This idea led John Jelley to contact Neil Porter in Dublin and F Graham Smith at the Cavendish Laboratory in Cambridge (who was about to move to Jodrell Bank) with the suggestion of using a small array of Geiger counters to trigger radio detection of air showers, taking advantage of the relatively radio-quiet zone at Jodrell Bank. Some of this work has been reported in reviews by Weekes (2001) and Fegan (2012). The major review by Allan (1971) also described the results from a variety of experimental groups including those at Harwell, Dublin, Haverah Park (Leeds), Moscow, Karkov, Bologna, Penticton, Bolivia and Australia.

The radio spectrum is rather crowded at wavelengths of a few metres. Because short pulses from the shower were expected, then a large bandwidth was needed. It was decided to use the frequency of $44 \mathrm{MHz}$, in the band used by the BBC TV video signal. Though this would mean operation during the daytime was not possible, the transmitters were turned off between the hours of 00:00 and 08:00 and so the nights were clear and free of interference. Electronic amplifiers covering the frequency band were also available from the Cavendish radio astronomy group. A suitable field big enough for a small particle detector array and antennas was found on the Jodrell Bank site, together with a hut to house the equipment and researchers. This is known as Blackett's Hut (figure 1) and had been built in 1948 free of magnetic materials to ........ allow Patrick MS Blackett to allow Patrick MS Blackett to
investigate the production of a magnetic field from a rotating massive body, in this case $15.2 \mathrm{~kg}$ of gold that he had borrowed from a bank, in 1949 (Lovell 1975).

The antenna was constructed in the summer of 1964 by Rob Porter, a graduate student at the University of Manchester. It comprised a $6 \lambda$ by $6 \lambda$ array of full-wave dipoles connected by open wire transmission line to a preamplifier and then by coaxial cable to the equipment in Blackett's Hut (Porter 1967). The particle detector array consisted of three trays of Geiger counters spaced $50 \mathrm{~m}$ apart in an equilateral triangle adjacent to the antenna, as shown in figure 2.

Coincident pulses from the counters triggered an oscilloscope fitted with a recording camera. The radio signals from the antenna were delayed to allow for delays in the triggering system, filtered, amplified and the power measured before being displayed on the oscilloscope. Trevor Weekes, then a graduate student at the University College, Dublin, had built the Geiger array and came over to help with running the experiment, which formed part of his PhD thesis. Trevor was lucky 


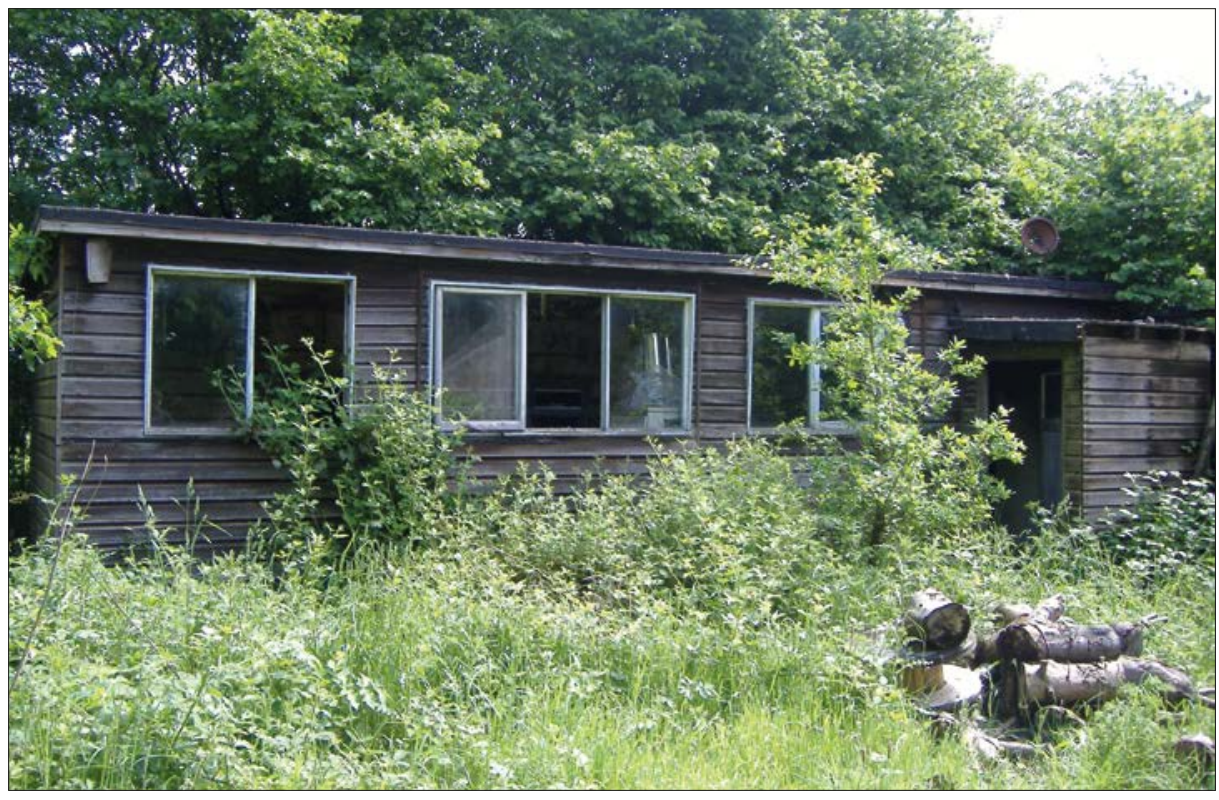

1 Blackett's Hut in the summer of 2008, now in a rather sorry state, though still containing evidence of the work done there in 1966 with a sketch of the particle detector array on the inside wall. In the 1960s, the hut contained the electronics and recording oscilloscope.

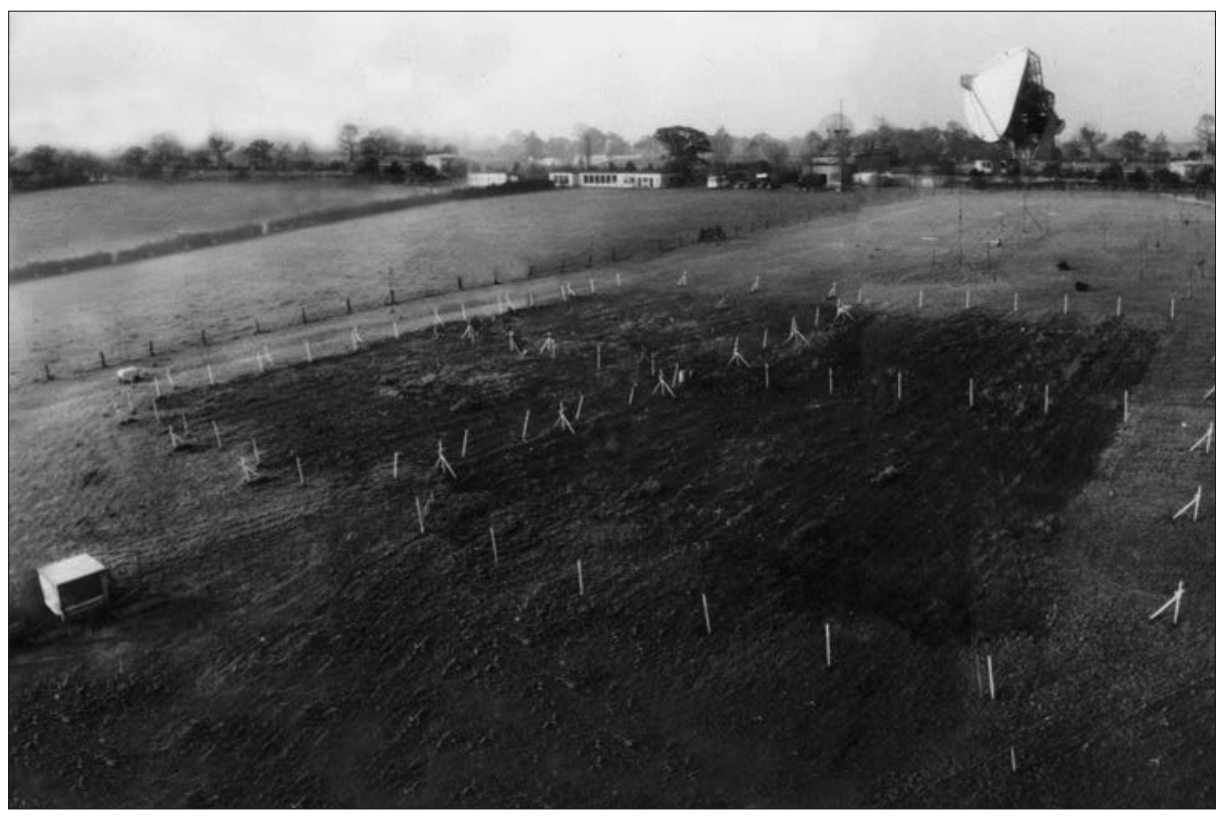

2 Layout of the equipment looking west, from a montage by RA Porter (1967). The dark area shows the position of the large array. The north-south corner reflector can be seen to the west of the array, in front of the Mk2 radio telescope. The white boxes contained the Geiger counters.

\section{A radio event at}

$44 \mathrm{MHz}$ as recorded

during the first

experiment in 1965

The sine wave was

used as a timing check.

The hodoscope - an

array of light-emitting

diodes - at bottom

left showed which

detectors were

triggered. The clock

and timing waveform

were not used in later

experiments.

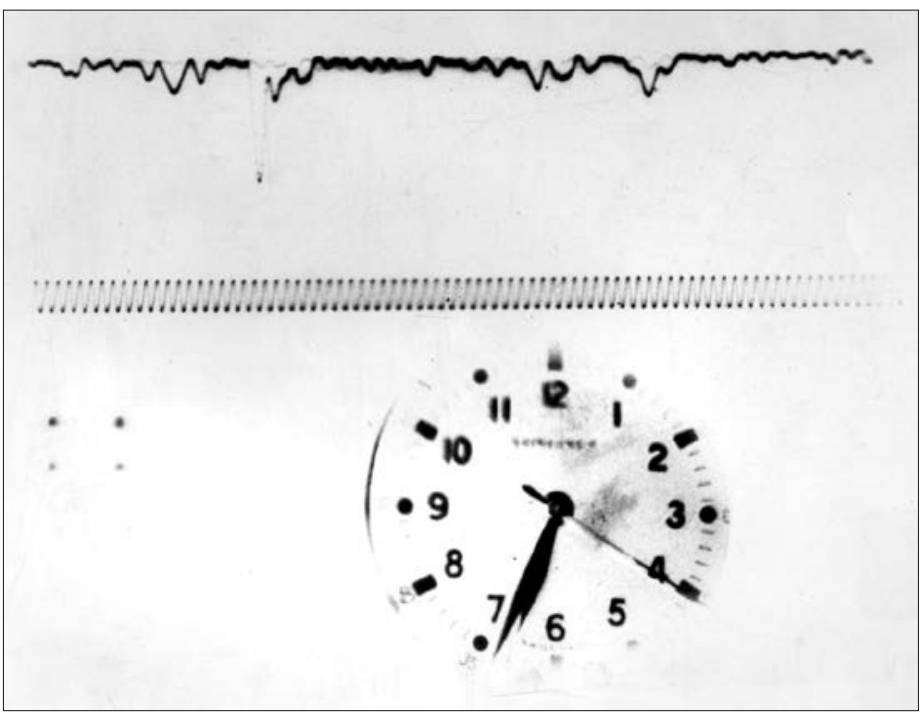

enough to find a large radio event on the fifth frame of the film used on the first successful night of operation 20 August 1964, as he recounted amusingly in Weekes (2001). Operations continued until March 1965, producing 4500 triggered events with 11 clearly visible pulses in the expected delay window (figure 3). Results were published in Nature (Jelley et al. 1965) and in Nuevo Cimento (Jelley et al. 1966).

\section{Radiation mechanisms}

Further experiments at Jodrell Bank concentrated on determining the mechanism for the radiation. Jelley listed possible mechanisms in 1965, identifying weak effects from: bremsstrahlung from shower particles via the Coulomb field of atoms in the air; bremsstrahlung from low-energy electrons ( $\delta$-rays); transition radiation as the shower enters the ground; induction effects; and molecular transitions in the radio band. He considered charge separation giving rise to dipole Cherenkov radiation and synchrotron radiation from electrons curving in the Earth's magnetic field more important, as well as Cherenkov radiation from a charge excess as suggested by Askaryan (1962). Kahn and Lerche (1966) were the first to show the relative importance of the magnetic field in determining the intensity of the radio pulse. Three components of the emission were identified: the current mechanism as the electrons are deflected by the Earth's field (essentially the synchrotron mechanism); the dipole Cherenkov radiation; and the charge excess Cherenkov. The current (synchrotron) mechanism was found to give the most intense pulses. Colgate (1967) also calculated pulse intensities by equating the momentum carried by the pulse to that lost by the particles being deflected by the magnetic field. Fujii and Nishimura (1969) extended the Kahn and Lerche theory to include a finite shower thickness and Spencer (1970) included the effects of an idealized lateral distribution in the shower front. Modern calculations use the much more realistic Monte Carlo cascade models of the development of cosmic-ray air showers (CORSIKA) and microscopic models for the radio emission.

The Earth's magnetic field has a dip angle (inclination) of $68^{\circ}$ at Jodrell Bank. Because the radiated pulse amplitude is proportional to the sine of the angle between the electron trajectory and the field, we would expect a stronger signal from showers arriving from the north than the south. A right-angled double corner reflector was constructed by Porter using a vertical reflector oriented east-west with north and south reflectors fed by four half-wave dipoles separated by 0.5 wavelengths. An analysis of several thousand 
events showed that there was no significant difference between the strengths of pulses from the north or the south, though the results were consistent with an equal contribution from the charge excess and geomagnetic mechanisms (Porter et al. 1967). Porter also performed a polarization experiment in which the south side of the main array was rotated by $90^{\circ}$ with no significant effect, even after 50 days of measurements.

As a result of these inconclusive experiments, it was decided to build more sensitive apparatus by using a phased radio array with twin beams, directed parallel and perpendicular to the Earth's magnetic field. It was also necessary to find the general direction of arrival of the air shower, and so the Geiger counters were replaced with scintillators, as shown in figure 4, and fast timing used to select showers coming from the north or south. In 1966, Tony Bray was given the task of designing and implementing the radio array, now operating at $42 \mathrm{MHz}$, while Porter built the trigger and timing electronics. The experiment ran in 1967, producing 2000 events. More strong events were seen in the northern beam, perpendicular to the magnetic field, showing that the geomagnetic effect predominates, though there was some evidence that weaker showers had an equal contribution from the charge excess mechanism (Bray 1969).

\section{The radio spectrum}

Ralph Spencer's interest in radio emission from cosmic rays was kindled by a talk given late in 1965 by Jelley to physics undergraduates at the University of Birmingham. He joined the new MSc course in Radio Astronomy at Jodrell Bank in October 1966. He chose cosmic-ray work for his thesis project and then for his PhD. To his

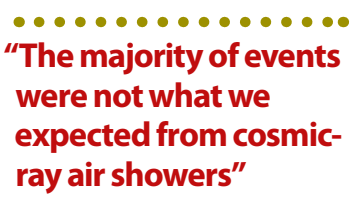

4 Diagram of a scintillator designed at AERE Harwell used for all experiments after the summer of 1966. The liquid scintillator consisted of medicinal liquid paraffin with p-terphenyl and POPOP as the active ingredients. Highvoltage supplies in Blackett's Hut were connected via coaxial cable to the scintillators, as were the electronics to form event triggers.

giving a rough indication of the location of the shower. Shower sizes were estimated to be a few multiples of $10^{5}$ particles for the triangle and square arrays, and $3 \times 10^{6}$ particles for the whole array, corresponding to a primary energy between $10^{15}$ and $10^{16} \mathrm{eV}$. Figure 5 shows the layout of the equipment in the field at this time.

The aim of the experiment was to investigate the radio spectrum of the pulses. We realized that coherence would be lost as the wavelength approached the shower thickness, meaning that the radiation intensity would be expected surprise, the first duty of a new student was to have a fitting for wellington boots and then to dig post holes.

The particle detector array covered the whole of the field (figure 5), and the outputs of the photomultipliers were connected back to the discriminators and coincidence unit in Blackett's Hut through $300 \mathrm{~m}$ of coaxial cable. The scintillators were arranged as two equilateral triangles of side $50 \mathrm{~m}$ and a central square of the same half-diagonal as the distance from a vertex to the centroid of the triangles. The central square was used for the fast timing. Slow coincidences $(2 \mu \mathrm{s})$ were also used, and a trigger was produced if either of the triangles or the square array produced a coincidence. The trigger combination was displayed on a hodograph array of lightemitting diodes in the oscilloscope camera, to be much lower at, say, $300 \mathrm{MHz}$ than at $40 \mathrm{MHz}$. There would also be a more rapid fall off with frequency at large lateral distances from the shower core as a result of geometric effects. We needed to confirm this and perhaps find the optimum frequency for detecting large showers.

The first task was to design a receiver for use at $105 \mathrm{MHz}$, just above the FM broadcast band, again working on the assumption that it would be quiet at night! A small $2 \lambda$ by $2 \lambda$ array of half-wave dipoles was built. Later experiments at $240 \mathrm{MHz}$ and $408 \mathrm{MHz}$ used a $9 \mathrm{~m}$ diameter parabolic wire-mesh dish originally built for a demonstration at the Festival of Britain in 1951. Calibration was via careful measurement of the noise performance of the receivers including the effects of the Milky Way galaxy, but the antenna gains were estimated from theory. We examined 4000 photographs but did not see any strong pulses. Most of the analysis was done by pulse counting, where the position of the strongest signal in each trace was noted. The most common position was at the expected time delay along the trace. The results (Spencer 1969,1970 ) showed a steep spectrum for the emission with the field strength of the pulses falling off as $E \sim f^{-1}$, after correcting for bandwidth, which is in agreement with later work. There was some evidence that the more distant showers were relatively weaker at the higher frequencies, though indeterminate shower size selection effects were important. What was clear is that the signal-to-noise ratio was close to optimum at $\sim 30-40 \mathrm{MHz}$, clearly justifying our original assumptions.

\section{The Hafren experiment}

The detection of large extensive air showers by radio means alone poses a number of problems. Observers need to be able to detect broadband pulses, in a heavily used frequency band, and to be able to find the direction to a reasonable accuracy. A radio-only timing experiment was needed in a radio-quiet site away from populated areas. In 1969, the Jodrell group decided to try an experiment using four broadband conical antennas operating between 30 and $60 \mathrm{MHz}$, and to trigger an oscilloscope and recording camera using coincidences between pulses from the spaced antennas. The signals from each antenna were 
delayed in steps of $1 \mu \mathrm{s}$, and the oscilloscope triggered four times in order to show the signal from each antenna in turn. The relative timing of short pulses could then give an indication of the direction to an accuracy of $5-10^{\circ}$ in azimuth and $\sim 10^{\circ}$ in elevation. A camera with continuously moving film recorded the oscilloscope display. The running speed and film cassette capacity allowed unattended operation for more than a week. A two-channel chart recorder monitored the coincidence unit output and the automatic gain control (AGC) output of one channel.

After commissioning the antennas and electronics at Jodrell Bank, the equipment was moved to a clearing in Hafren Forest (figure 6), on the side of Plynlimmon in midWales - which certainly counted as a remote site, requiring a five-hour journey from Cheshire in a four-wheel-drive vehicle.

The equipment was run for two years, mostly through the summer, after operations in the first year showed that access in the winter on forest tracks was somewhat limited. The equipment was checked and films collected once per week. Early results showed that the site was indeed relatively radio quiet with Band 1 TV signals from distant (Spanish TV) transmitters only being detected in anomalous ionospheric conditions. What was also clear was that there were several types of events, the majority not being the bandwidth-limited narrow pulses that we expected from cosmic-ray air showers. Interference levels, though much weaker than at the Jodrell Bank site, were nevertheless problematic (Porter et al. 1973). A comprehensive analysis was done by Chris Rapley for his MSc thesis (Rapley 1970).

Based on an estimated collecting area for the array of $\sim 2 \times 10^{4} \mathrm{~m}^{2}$, and an estimated detection threshold of $\sim 10^{17} \mathrm{eV}$, the anticipated event rate was $\sim 15$ air showers per week. This calculation was approximate, and did not take into account the sky-limited night-time variations of the automatic gain control, nor the reduction in gain and observing time lost through daytime TV transmissions and radio interference. The antenna polar diagrams with a half power point at approximately $35^{\circ}$ and a zenith null also reduced the sensitivity to air showers from high elevation angles.

\section{Puzzling pulses}

In practice, analysis of five weeks of data collected in the autumn of 1969 showed rates $\sim 10^{5}$ pulses per week, far more than expected. The events were found to fall into three general classes. The first dominated the records, with $\sim 8 \times 10^{4}$ counts per week. They were detected at high rates over long periods at night when the system sensitivity was highest, and more sporadically at

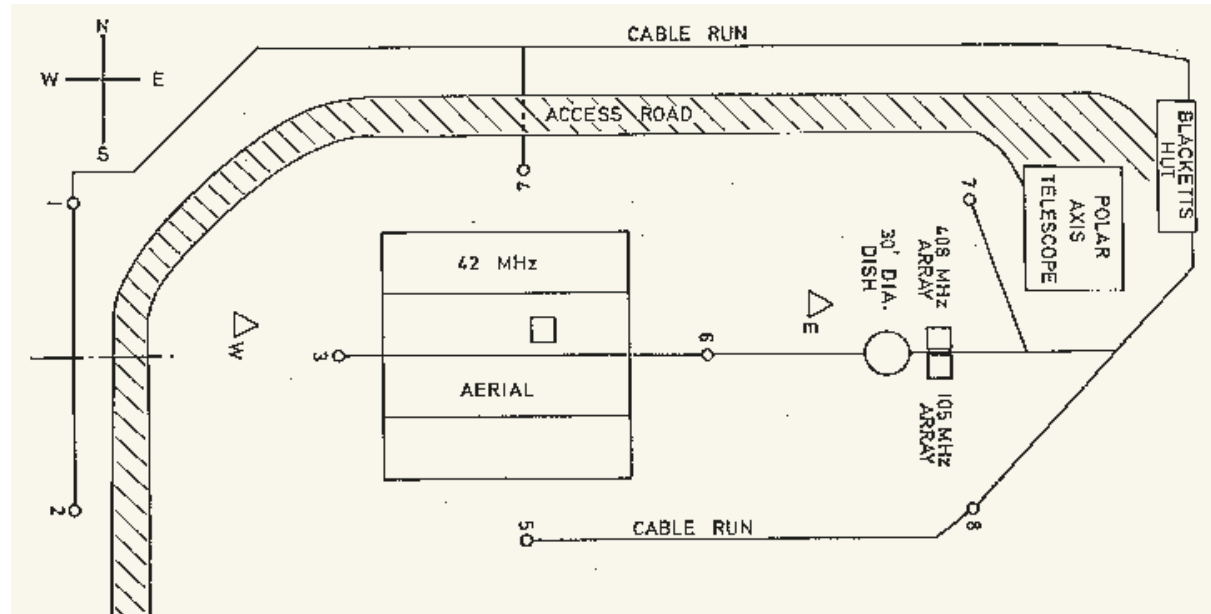

5 Arrangement of scintillators and radio aerials in the field adjacent to Blackett's Hut in 1966. The small circles denote the positions of the scintillators, with the triangle and square symbols representing the coincident trigger arrangements. The equilateral triangle arrays of detectors had spacings of $50 \mathrm{~m}$ on each side. The Polar Axis Telescope was used for lunar radar during these experiments.

other times. The pulses had characteristic shapes, with fixed but apparently impossible inter-channel delay times, except in one channel where the pulse delay was sporadic. This was puzzling, until it was realized that a source solution could be found within the array, coincident with the diesel generator to within a few metres. The sporadic pulses occurred in a channel with an antenna obscured from the generator by a ridge, modulating the system triggers by multipath or random detections. The interference commenced after a known problem with the generator, and could have been eliminated had the problem been picked up and understood during the deployment. The delay in the processing and analysis of the film records, plus the occurrence of the pulses at times when the system was unattended, militated against this.

The second class of events occurred in large-amplitude bursts of $\sim 1$ min duration during the working day. They occurred on average about 20 times a week, and contributed $\sim 2 \times 10^{4}$ counts to the total. Source directions were found to coincide within the uncertainties with local farms and tracks and a ploughed field lying between $1500-3000 \mathrm{~m}$ of the array, and were attributed to electrical interference from vehicles and machinery. Three bursts originated from the sky, one of which tracked from an elevation of 0 to $60^{\circ}$ in $\sim 30$ seconds; these were attributed to aircraft or helicopters.

The third class of events contributed on average 400 pulses per week, most of them in low rate $(<2$ per minute) groups of between 2 and 150 low-amplitude pulses, with random relative delays, mostly during the working day. These also were attributed to weak electrical interference. We also considered the possibility of picking up electrical discharges (St Elmo's fire)

from the pine needles of the forest, but a laboratory experiment gave a null result. No electrical storms were reported during the observing period.

One single bandwidth-limited pulse was detected on 31 August 1969 at 02.12UT and was attributed to an $\sim 10^{17} \mathrm{eV}$ air shower at $30^{\circ}$ elevation.

Based on this experience of the difficulties of running a remote site without the

ifficulties site, we and analysis of the results of the Hafren experiment, we decided to redeploy the array on site at Jodrell Bank. Improvements were made to the AGC and threshold discriminators, and the ability to operate with selectable three-fold rather than only four-fold coincidence introduced. We located one antenna in a small copse, and the others on open ground, allowing any effect of obscuration by trees to be investigated; none was found. However, the main innovation here was the development of a real-time display of source azimuth and elevation on the oscilloscope.

We obtained mean daily pulse totals an order of magnitude greater than at Hafren. However, a direct numerical comparison is misleading given the saturating effect of the $\mathrm{BBC} 1$ television signal during the day. In reality, the Jodrell environment was very much noisier. Approximately onethird of each day's total pulses occurred between the hours of 3.30 and 7.30UT, when the system was still sky- rather than television-limited. The preceding four hours could be relatively free of interference, containing only $\sim 25$ pulses, although on some nights high levels of interference continued non-stop.

For operational reasons and lack of time, it was not possible to develop and analyse the film records. But visual analysis of the oscilloscope display showed correlations of 

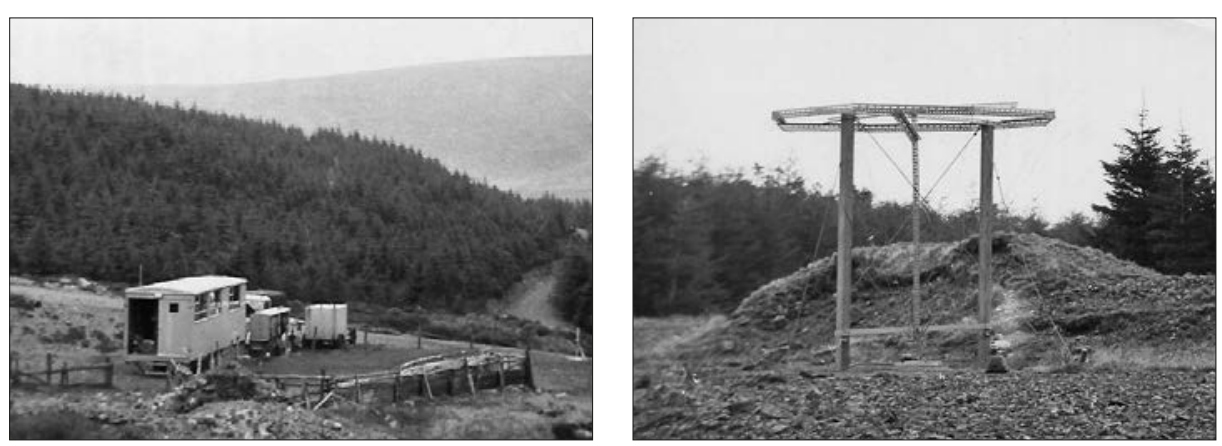

6 The Hafren experiment. (Left) The equipment trailer on the site labelled Sheep Dip on the Ordnance Survey map. (Right) One of the four monopole conical antennas. The radiating element was a set of wires (not visible in the picture) connected from the upper frame down to a balun transformer on the ground.

pulses with passing vehicles and as many as $90 \%$ of events at zero elevation.

More interesting were signals observed during a series of heavy thunderstorms, in which pulse rates of 100 s per second were observed in multiple bursts lasting several seconds with source directions associated with visible thunderheads. Most bursts terminated in a sudden rapid change of direction. On occasion, several sources were detected simultaneously and were seen to streak in different directions across the sky. One researcher's noise is another's signal, and it was concluded that while atmospheric electrical storms place a limit on air-shower observations, they provide an interesting source of information in their ................... "There have been major developments in the theory of the radio emission mechanism"

\section{A renaissance}

Active work on the radio emission from cosmic rays diminished after 1975, with few papers published in the following 30 years (Weekes 2001, Huege 2016). But a November 2000 meeting in Los Angeles on the radio detection of highenergy particles (Salzberg \& Gorham 2001) led to a revival of interest in the subject (see review by Huege 2016). The own right. We note that the atmospheric electric field strongly affects the radio emission from extensive air showers (Mandolesi et al. 1974, Buitink et al. 2007) - indeed air showers may even initiate lightning strikes (Dubinova et al. 2015). We did, however, notice that the electromagnetic pulse from nearby lightning strikes triggered the particle detector array at Jodrell Bank through pick-up on the cables, and produced detectable signals in the radio receivers, so the situation may be confused.

The outcome of the Hafren experiment was to underscore the difficulties of achieving interference-free observations even at a remote site, but also to demonstrate that, even at a much noisier site such as Jodrell, use of digital techniques made sophisticated experiments possible (Falcke \& Gorham 2003) in which signals from many antennas could be processed. The new experiments include CODALEMA (Ardouin et al. 2005), LOPES (Falcke et al. 2005), the Auger Engineering Radio Array (AERA, Schulz 2015), LOFAR (Schellart et al. 2013) and Tunga-Rex (Bezyazeekov et al. 2015).

There have also been major developments in the theory of the radio emission mechanism, with microscopic approaches following the tracks of individual particles. The geosynchrotron approach has been found to be incorrect because the particle tracks are relatively short as a result of interaction with air molecules. Modern codes such as CoREAS and ZHSAires use the results from Monte Carlo shower simulations (reviewed by Huege 2016) and show good agreement with experimental results.

Over the past decade, the radio technique has become a mainstream addition to particle arrays for the study of cosmic rays with energies greater than $10^{17} \mathrm{eV}$. Since the radio emission depends on the longitudinal dependence of the shower, radio methods can be used to indicate the composition of the primary particles (e.g. Buitink et al. 2016). The fact that radio antennas and receivers are relatively inexpensive means that large areas can be covered even though the radio footprint of the showers is only a few hundred metres in diameter. Perhaps the most exciting prospect is the measurement of the composition of cosmic rays of energy $10^{17}-10^{18} \mathrm{eV}$ with high precision using the SKA Low Frequency Array to be built in a radio quiet zone in Western Australia (Huege et al. 2017). Development of a scintillator particle detector to trigger data collection from the many antennas in the array is currently taking place at Jodrell Bank.

\section{Conclusion}

Many of the basic characteristics of radio emission from extensive air showers were found by the early research, through sometimes heroic efforts. Now much has changed, mainly as a result of the use of digital technology, which has enabled automatic analysis from many antennas and realistic theoretical modelling.

Recent experiments have tended to concentrate on showers with energies of $>10^{17} \mathrm{eV}$, well above the threshold for the experiments in the 1960s. The radio signals are strong and easily detectable by single dipole antennas. Our early experiments therefore suffered from a lack of suitably strong events - which explains why so many triggers had to be detected before events with good signal-to-noise ratios were found. The accuracy of modern measurement is much superior to that in the 1970s and the use of radio has now become an essential addition to the armoury of techniques for the study of cosmic rays.•

\section{AUTHORS}

Ralph Spencer, School of Physics and Astronomy, The University of Manchester, UK. Chris Rapley, Department of Earth Sciences, University College London, UK.

\section{ACKNOWLEDGMENTS}

The authors thank Sir Francis Graham-Smith and Justin Bray for valuable comments on the manuscript.

\section{REFERENCES}

Allan HR 1971 Progress in Elementary Particle and Cosmic Ray Physics $\mathbf{X} 171$

Ardouin D et al. 2005 Nucl. Instr. Meth. A 555148 Askaryan GA 1962 Sov. Phys. J. E. T. P. 14441 Bezyazeekov PA et al. 2015 Nucl. Instr. Meth. A

\section{9}

Blackett PMS \& Lovell ACB 1941 Proc. Roy. Soc. 177183

Bray A 1969 Nature 223723

Buitink S et al. 2007 Astron. \& Astrophys. 467385 Buitink S et al. 2016 Nature 53170

Colgate SA 1967 J. Geophys. Res. 724869

Dubinova A et al. 2015 Phys. Rev. Lett. 115015002 Falcke H \& Gorham PW 2003 Astropart. Phys. 19477

Falcke H et al. 2005 Nature 435313

Fegan D 2012 Nucl. Instr. Meth. A 662 S2

Fujii M \& Nishimura J 1969 Proc. IUAP Conf. Cos. Rays. Budapest 69

Gorham P 2001 in eds Salzberg D \& Gorham P AIP Conf. Proc. $\mathbf{5 7 9} 253$ Hess VE 1911 PhysikZ 12998
Huege TC 2016 Phys. Rep. 620

Huege TC et al. 2017 EPJ Web. Conf. 13502003 Jelley JV 1958 Cerenkov Radiation and Its Applications (Pergamon Press)

Jelley JV et al. 1965 Nature 205327

Jelley JV et al. 1966 Nuovo Cim. 46649

Kahn FD \& Lerche I 1966 Proc. Roy. Soc. A 289206

Lovell B 1968 The Story of Jodrell Bank (Oxford

University Press)

Lovell B 1975 Biogr. Mems Fell. R. Soc. 211

Mandolesi N et al. 1974 J. Atmos. Terr. Phys. 36 1431

Patrignani C et al. (Particle Data Group) 2016 The Review of Particle Physics Chin. Phys. C 40

100001 chapter 29

Porter RA 1967 unpublished MSc thesis (University of Manchester)
Porter RA et al. 1967 Nature 213110

Porter RA et al. $1973 \mathrm{~J}$. Atmos. Terr. Phys 351421 Rapley C 1970 unpublished MSc thesis (University of Manchester)

Salzberg D \& Gorham PW (eds) 2001 Radio Detection of Higher Energy Particles AIP Conf Proc 579 Schellart P et al. 2013 Astron. \& Astrophys. 560 A98 Schulz J for the Pierre Auger Collaboration 2015 Proc. 34th ICRC The Hague, The Netherlands POS(ICRC) 615 Spencer RE 1969 Nature 222460 Spencer RE 1970 unpublished PhD thesis (University of Manchester) Weekes TC 2001 in Radio Detection of Higher Energy Particles eds Salzberg D \& Gorham P AIP Conf.Proc. 5793 